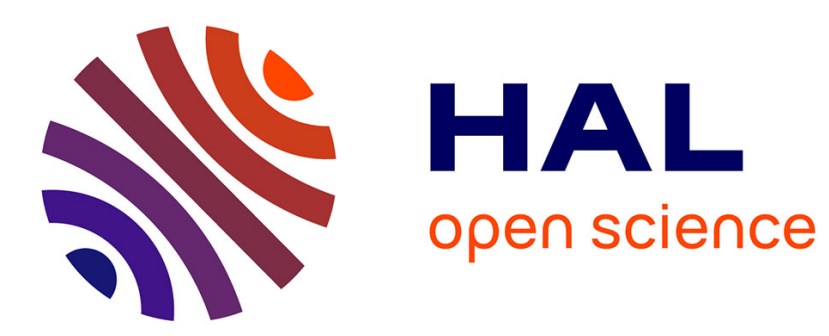

\title{
Semi-Blind Cancellation of IQ-Imbalances
}

\author{
Matthias Hesse, Marko Mailand, Hans-Joachim Jentschel, Luc Deneire, \\ Jerome Lebrun
}

\section{To cite this version:}

Matthias Hesse, Marko Mailand, Hans-Joachim Jentschel, Luc Deneire, Jerome Lebrun. Semi-Blind Cancellation of IQ-Imbalances. IEEE International Conference on Communications, May 2008, Beijing, China. pp.5023-5027. hal-00272886v2

\section{HAL Id: hal-00272886 https://hal.science/hal-00272886v2}

Submitted on 17 Jun 2008

HAL is a multi-disciplinary open access archive for the deposit and dissemination of scientific research documents, whether they are published or not. The documents may come from teaching and research institutions in France or abroad, or from public or private research centers.
L'archive ouverte pluridisciplinaire HAL, est destinée au dépôt et à la diffusion de documents scientifiques de niveau recherche, publiés ou non, émanant des établissements d'enseignement et de recherche français ou étrangers, des laboratoires publics ou privés. 


\title{
Semi-Blind Cancellation of IQ-Imbalances
}

\author{
Matthias Hesse, Student Member, IEEE, Marko Mailand, Student Member, IEEE, Hans-Joachim Jentschel, \\ Senior Member, IEEE, Luc Deneire Member, IEEE and Jérôme Lebrun
}

\begin{abstract}
The technical realization of modern wireless receivers yields significant interfering IQ-imbalances, which have to be compensated digitally. To cancel these IQ-imbalances, we propose an algorithm using iterative blind source separation (IBSS) as well as information about the modulation scheme used (hence the term semi-blind). The novelty of our approach lies in the fact that we match the nonlinearity involved in the IBSS algorithm to the probability density function of the source signals. Moreover, we use approximations of the ideal non-linearity to achieve low computational complexity. For severe IQ-mismatch, the algorithm leads to $0.2 \mathrm{~dB}$ insertion loss in an AWN channel and with 16-QAM modulation.
\end{abstract}

\section{Index Terms}

Semi-blind source separation, IQ-imbalances, zero-IF receiver

\section{INTRODUCTION}

I $\mathrm{N}$ modern mobile communication systems, the amount of analog components is minimised, in order to reduce both the cost and the space of transceivers. A well known receiver architecture which follows this principle is the zero-IF receiver [1], which enables efficient analog front-ends, but leads to non perfect orthogonality and amplitude matching between the In-phase and Quadrature baseband signals (IQ-mismatch).

The zero-IF receiver, which is also know as direct-conversion or homodyne receiver, converts the bandpass signal directly to the baseband without using an intermediate frequency (IF). For handling complex modulations, the down conversion in the I- and Q-path is done separately with a phase offset of $90^{\circ}$. Due to tolerances in analog components, there is an amplitude mismatch as well as a phase error, which culminates in a mixture of the I- and Q-components.

Estimation of the IQ-imbalance can be done with a pilot [2] or in a decision-directed way [3], [4]. Thereupon, the respective compensation is now based on this estimation. Direct compensation can be done blindly by using signal separation techniques to separate the I- and Q-signals [5]-[7]. This technique circumvents the use of a pilot tone and the delay due to decisions. Under the assumption that the I- and Q-signals are statistically independent, Valkama [8], [9] showed how to separate this mixture with an equivariant adaptive separation via independence (EASI) algorithm [10].

The statistical independence hypothesis does not catch the specific nature of the signals, i.e. the fact that the signals are modulated data. We take this additional knowledge into account and exploit it within the blind source separation (BSS) to enhance the performance of the EASI algorithm as follows. To ensure statistical independance of the I- and Q-signals, the IBSS algorithm diagonalizes a correlation matrix between a linear transform and a non-linear transform of the received signal. By matching this non-linear transform to a specific function of the pdf of the source, the separation is optimized.

The paper is organized as follows. In Section II, we describe the model which characterizes the IQ-imbalances and the separation system. In Section III, we propose the pdf-dependent non-linearities and provide a simplification in order to reduce the computational complexity. In Section IV, we compare the performance of the proposed non-linearities, three widely used non-linearities and the EASI algorithm. In Section V, we draw the main conclusions and perspectives.

M. Hesse, L. Deneire and J. Lebrun are with the Laboratoire I3S, Algorithmes/Euclide-B, 2000, Route des Lucioles, BP121, 06903 Sophia Antipolis-Cedex, France; e-mail: \{hesse,deneire,lebrun\}@i3s.unice.fr;

M. Mailand and H.-J. Jentschel are with the Institute of Traffic Communications Engineering, "Friedrich List" Faculty of Transport and Traffic Sciences, Dresden University of Technology, Hettnerstraße 3, 01062 Dresden, Germany; e-mail: Marko.Mailand@ieee.org, Hans-Joachim.Jentschel@tu-dresden.de; .

The work of M. Hesse is supported by the EU by a Marie-Curie Fellowship (EST-SIGNAL program : http://est-signal.i3s.unice.fr) under contract No MEST-CT-2005-021175. 


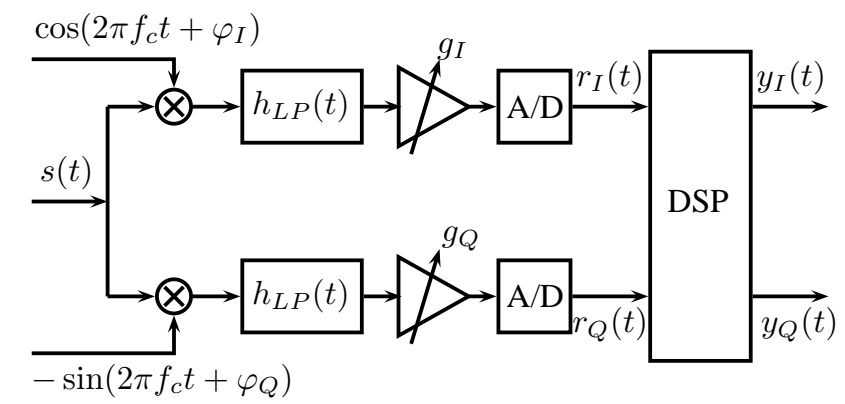

Fig. 1. Block diagram of a zero-IF receiver with imbalances of phase $\left(\varphi_{I}, \varphi_{Q}\right)$ and amplitude $\left(g_{I}, g_{Q}\right)$ in I- and Q-path

\section{SYSTEM MODEL}

In this section, we describe the two main parts of our model. Each part on its own, is well known in the corresponding field of research.

\section{A. Mixture Model}

The received signal

$$
\begin{aligned}
s(t) & =\operatorname{Re}\left\{\underline{d}(t) \exp \left(j 2 \pi f_{c} t\right)\right\}+n(t) \\
& =\operatorname{Re}\{\underline{d}(t)\} \cos \left(2 \pi f_{c} t\right)-\operatorname{Im}\{\underline{d}(t)\} \sin \left(2 \pi f_{c} t\right)+n(t)
\end{aligned}
$$

is split by the zero-IF receiver into the orthogonal I-component $r_{I}(t)$ and Q-component $r_{Q}(t)$ by multiplying with $\cos \left(2 \pi f_{c} t\right)$ and $-\sin \left(2 \pi f_{c} t\right)$, respectively. In this ideal case, the I-path contains the real part of the transmitted symbol $\operatorname{Re}\{\underline{d}(t)\}=$ $d_{I}(t)$, some high frequency (HF) components and noise $n(t)$, the Q-path includes the imaginary part $\operatorname{Im}\{\underline{d}(t)\}=d_{Q}(t)$, HF components and also some noise $n(t)$. The HF components are removed by a low pass filter $h_{L P}(t)$ being assumed to be ideal.

The imperfection of the local oscillators (LO) yields phase errors (denoted as $\varphi_{I}$ for the I-path and $\varphi_{Q}$ for the Q-path (Figure 1) which are assumed to be time invariant. Due to this maladjustment, the Q-component is interfered by the I-signal and vice versa. Furthermore, due to production imperfections, the mismatches in the circuitry cause amplitude imbalances. We model these as an imbalance in gain $g_{I}$ and $g_{Q}$ (Figure 1) which is also assumed to be invariant in time. Having defined these impairments, we are able to compute the disturbed I- and Q-signal by

$$
\begin{aligned}
r_{I}(t) & =g_{I} \cos \left(\varphi_{I}\right) d_{I}(t)-g_{I} \sin \left(\varphi_{I}\right) d_{Q}(t) \\
r_{Q}(t) & =-g_{Q} \sin \left(\varphi_{Q}\right) d_{I}(t)+g_{Q} \cos \left(\varphi_{Q}\right) d_{Q}(t) .
\end{aligned}
$$

A common assumption in the literature is that $\varphi_{I}$ is zero and $\varphi_{Q}$ is only the phase difference between I- and Q-path. That means that the I-path is perfectly synchronised. In this paper, we don't make this assumption. Simulations in section IV show that small synchronisation errors are also compensated.

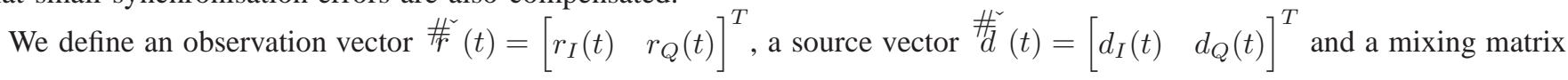

$$
\mathbf{H}=\left[\begin{array}{ll}
h_{11} & h_{12} \\
h_{21} & h_{22}
\end{array}\right]=\left[\begin{array}{cc}
g_{I} \cos \left(\varphi_{I}\right) & -g_{I} \sin \left(\varphi_{I}\right) \\
-g_{Q} \sin \left(\varphi_{Q}\right) & g_{Q} \cos \left(\varphi_{Q}\right)
\end{array}\right] .
$$

The mixture model is now given by

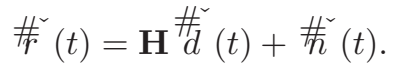

The task of the BSS algorithm is to estimate the separation matrix $\mathbf{W}_{*}=\mathbf{H}^{-1}$ such that the separated vector $\mathbb{H}^{2}(t)=$

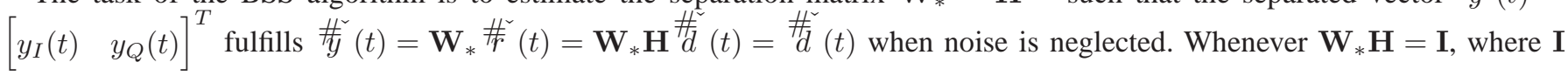
is a $2 \times 2$ identity matrix, the IQ-imbalances are cancelled. 


\section{B. Separation System}

The iterative inversion (II) [11], [12] is an optimization algorithm with a maximum likelihood (ML) criterion [13] (or equivalently, the mutual information criterion [14] and the INFOMAX criterion [15]). The purpose of these criteria is to restore the statistical independence of the source signals. Therewith the data sent over the I- and Q-path have to be statistically independent.

The optimization of all three criteria $\Psi$ results in the condition for independence:

$$
\left.\frac{\partial \Psi}{\partial \mathbf{W}}\right|_{\mathbf{W}=\mathbf{W}_{*}}=\mathbf{R}_{\psi\left(y^{-}\right) H^{-}}-\left.\mathbf{W}^{-T}\right|_{\mathbf{W}=\mathbf{W}_{*}}=0
$$

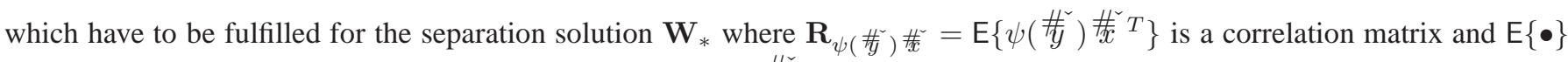

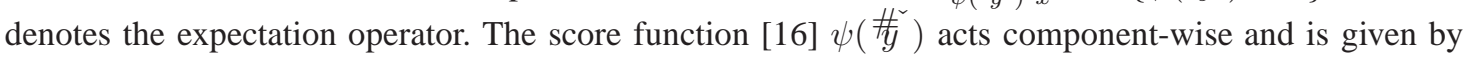

$$
\begin{aligned}
\psi\left(\text { H }^{\check{y}}\right) & =\left[\begin{array}{ll}
-\mathrm{d} \ln p_{\hat{d}_{Q}}\left(y_{Q}\right) / \mathrm{d} y_{Q} & -\mathrm{d} \ln p_{\hat{d}_{I}}\left(y_{I}\right) / \mathrm{d} y_{I}
\end{array}\right]^{T} \\
& =\left[\begin{array}{ll}
-p_{\hat{d}_{Q}}^{\prime}\left(y_{Q}\right) / p_{\hat{d}_{Q}}\left(y_{Q}\right) & -p_{\hat{d}_{I}}^{\prime}\left(y_{I}\right) / p_{\hat{d}_{I}}\left(y_{I}\right)
\end{array}\right]^{T}
\end{aligned}
$$

where $p_{\hat{d}_{i}}\left(y_{i}\right)$ is the estimated pdf of the source signal $d_{i}$ and $p_{\hat{d}_{i}}^{\prime}\left(y_{i}\right)$ is its first derivation.

In [11], equation (5) is solved numerically with a quasi-Newton algorithm. This algorithm approaches the separation solution $\mathbf{W}_{*}$ iteratively as

$$
\mathbf{W}^{(n+1)}=\mathbf{W}^{(n)}-\mu^{(n)}\left(\mathbf{R}_{f\left(\frac{n}{3}\right) g(\text { 龙 })}^{(n)}-\mathbf{I}\right) \mathbf{W}^{(n)} .
$$

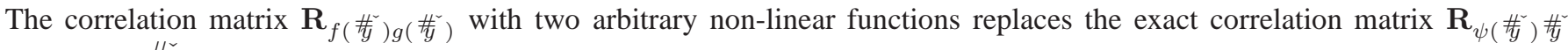
because $\psi\left(\frac{H_{y}}{)}\right)$ is unknown if the separation is completely blind. However, in communication systems the modulation is usually known in advance and therewith the pdf of the source. This a-priori knowledge can be used to determine $\psi($ 㳻 $)$ (section IIII)

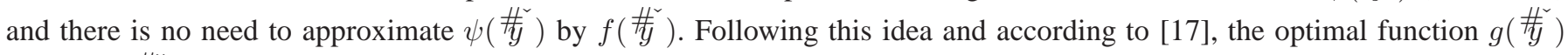
is simply $\#^{2}$.

In [11], two methods are proposed for estimating $\mathbf{R}_{\psi\left(\frac{1}{y}\right)}^{(n)}$ 䓔. The first one uses a single sample to estimate the correlation. This requires the least calculation effort and causes no additional delay. But, due to the bad estimation performance, the convergence behavior is degraded. The second technique averages the correlation by using a long fixed block (assuming stationarity and ergodicity), which leads to better estimation performance, at the cost of computational complexity and additional delay. In our approach, the delay and complexity are kept at a minimum by using a sliding window block of size $L$, enabling very small delay and low complexity by resorting to an adaptive implementation. Moreover, the block size $L$ can be adjusted to optimize performance.

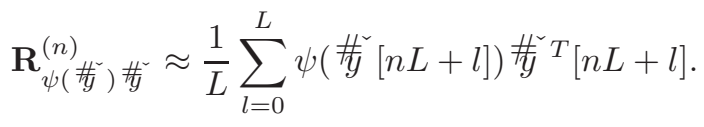

Finally, the convergence characteristics of the iterative inversion algorithm (7) are determined by the step size

$$
\mu^{(n)}=\frac{\eta}{1+\eta \| \mathbf{R}_{f\left(\frac{\left.H^{\prime}\right) g\left(\frac{1}{y}\right)}{(n)} \|\right.}}
$$

It is defined in a way that prevents the estimation of the separation matrix $\mathbf{W}$ from crossing any discontinuities. This way, the convergence of the algorithm is assured (if $0<\eta<1$ ) (convergence to a stationary point, which is not guaranteed to be a separation solution). 


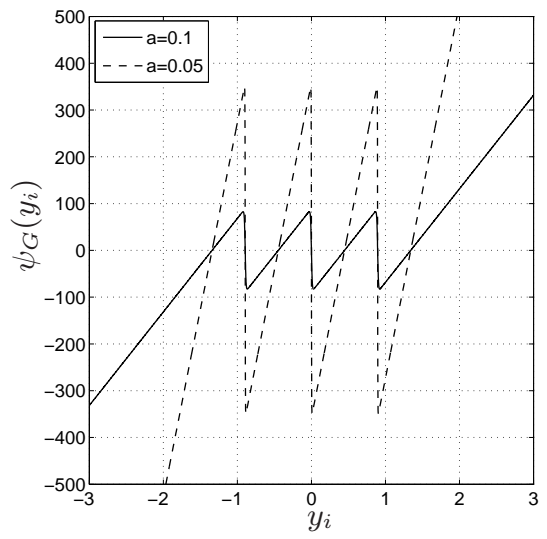

(a) Gauss NL

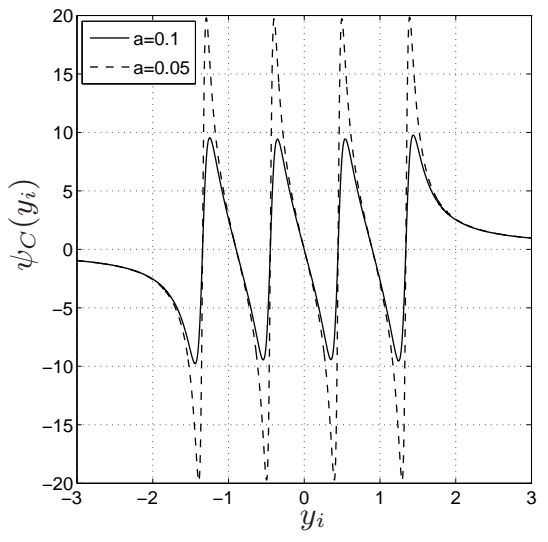

(b) Cauchy NL

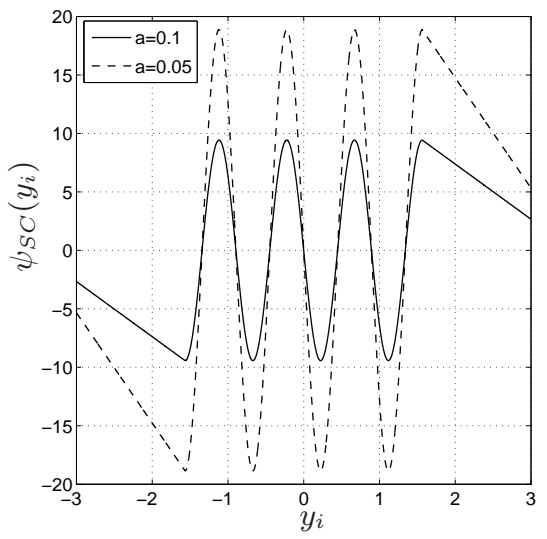

(c) Simplified Cauchy NL

Fig. 2. Different approximations of pdf dependent non-linearities to separate 4-PAM signals with unity energy.

For analyzing the quality of separation, we use the performance index (PI) [18]:

$$
\begin{array}{r}
P I^{(n)}=\sum_{i=1}^{N}\left(\sum_{j=1}^{N} \frac{\left|G_{i j}^{(n)}\right|^{2}}{\max \left\{\left|G_{i l}^{(n)}\right|^{2}\right\}}-1\right) \\
\ldots+\sum_{j=1}^{N}\left(\sum_{i=1}^{N} \frac{\left|G_{i j}^{(n)}\right|^{2}}{\max _{l}\left\{\left|G_{l j}^{(n)}\right|^{2}\right\}}-1\right)
\end{array}
$$

whereas $\mathbf{G}=\mathbf{W H}$ is the transfer function of the whole system. The performance index provides the quadradic distance between the transfer function and closest permutation of the unity matrix.

\section{NON-LINEARITIES}

In this section we propose three non-linearities $\psi\left(H^{-}\right)$that depend on the sources pdfs (like suggested by equation $(6)$ ), leading to a semi-blind approach (i.e. using more a-priori information). To obtain the semi-blind source separation we have to specify the pdf-dependent non-linearities suited to digital communication signals. Therefore we assume that the widespread $M^{2}$ quadrature amplitude modulation (QAM) is used, which corresponds to the use of $M$ pulse amplitude modulation (PAM) in I- and Q-path. The discrete pdf of a PAM signal $d_{i}$ is described by a sum of shifted and scaled Dirac impulses :

$$
p_{d_{i}}\left(y_{i}\right)=\frac{1}{M} \sum_{m=1}^{M} \delta\left(y_{i}-k(2 m-M-1)\right),
$$

with $M$ being the number of different amplitudes and

$$
k=\sqrt{\frac{M / 2}{\prod_{n=1}^{M / 2} 2 n-1}}
$$

as the half distance between two adjacent Dirac impulses. With this definition, unit variance of the source signals is guaranteed. Thus, it is not necessary to rescale the separated signals. The investigations of (6) and (11) show that the denominator of $\psi\left(y_{i}\right)$ is zero for most of the values of $y_{i}$. To avoid the non-defined expressions, the Dirac function is approximated by a Gaussian or a Cauchy distribution. 


\section{A. Gaussian approximation based non-linearity}

The limit of a Gaussian distribution for the variance at zero is the Dirac function. For $a=\sqrt{2} \sigma$ the pdf of the $M$-PAM source is approximated by

$$
p_{d_{i}}\left(y_{i}\right) \approx \frac{1}{a \sqrt{\pi}} \sum_{m=1}^{M} \exp \left(-\frac{y_{i}-k(2 m-M-1)}{a^{2}}\right) .
$$

With this approximation, we obtain a non-linearity which is defined for any $y_{i}$. Namely,

$$
\psi_{G}\left(y_{i}\right)=\frac{\sum_{m=1}^{M} \frac{2\left(y_{i}-k(2 m-M-1)\right)}{a^{2}} \exp \left(-\frac{\left(y_{i}-k(2 m-M-1)\right)^{2}}{a^{2}}\right)}{\sum_{m=1}^{M} \exp \left(-\frac{\left(y_{i}-k(2 m-M-1)\right)^{2}}{a^{2}}\right)} .
$$

Figure 2(a) shows the Gaussian non-linearity for different $a$. For decreasing $a$ the slope of the non-linearity increases and vice versa. Steep non-linearities lead to convergence problems, on the other hand less steep non-linearities denote a relatively large $a$ which is equivalent to a poorly approximated Dirac function. An optimized slope can be determined by red investigating the PI, depending on the parameter $a$ and $\eta$, with simulations. The optimization result is shown in Figure 3.

\section{B. Cauchy approximation based non-linearity}

Alike the Gaussian distribution, the limit of the Cauchy distribution for $a$ going to zero is the Dirac function, too. With the Cauchy distribution we get an approximated pdf as follows:

$$
p_{d_{i}}\left(y_{i}\right) \approx \frac{1}{\pi} \sum_{m=1}^{M} \frac{a}{a^{2}+\left(y_{i}-k(2 m-M-1)\right)^{2}} .
$$

The resulting approximation is used to calculate the Cauchy non-linearity:

$$
\psi_{C}\left(y_{i}\right)=-\frac{\sum_{m=1}^{M} \frac{2\left(y_{i}-k(2 m-M-1)\right)}{\left(a^{2}+\left(y_{i}-k(2 m-M-1)\right)^{2}\right)^{2}}}{\sum_{m=1}^{M} \frac{1}{a^{2}+\left(y_{i}-k(2 m-M-1)\right)^{2}}} .
$$

The Cauchy non-linearity is depicted in Figure 2(b). As for to the Gaussian non-linearity, the slope is optimized by computer simulations and the resulting optimized $a-\eta$-ratio is shown in Figure 3 .

\section{Simplified Cauchy non-linearity}

The two proposed non-linearities are precise but require a large computational effort. To alleviate this, we propose a sinusoidal approximation of the Cauchy non-linearity (indeed, a linear approximation of the Gaussian non-linearity would lead only to decorrelation and not statistical independence). The period of the sine wave is determined by the distance between two PAM symbols. A whole oscillation has the length of $2 k$ with zeros at the positions of the PAM symbols. The amplitude is fitted to the Cauchy non-linearity with $a=0.1$ which is given in Table 1 for different constellations. Due to the importance of the correct slope, a scaling factor $a^{\prime}=-A / 10 a$ corresponding to the other non-linearities is introduced. Thereby the amplitudes of the Cauchy and the simplified Cauchy non-linearity match for $a=0.1$. For the outer areas, linear functions are employed. We finally obtain the simplified Cauchy non-linearity expressed by:

TABLE I

MAXIMUM AMPLITUDE $A$ OF THE CAUCHY NON-LINEARITY FOR M-PAM

\begin{tabular}{ccccc}
\hline $\mathrm{M}$ & 4 & 8 & 16 & 64 \\
\hline $\mathrm{A}$ & 9.43 & 7.46 & 3.33 & 0.363 \\
\hline
\end{tabular}




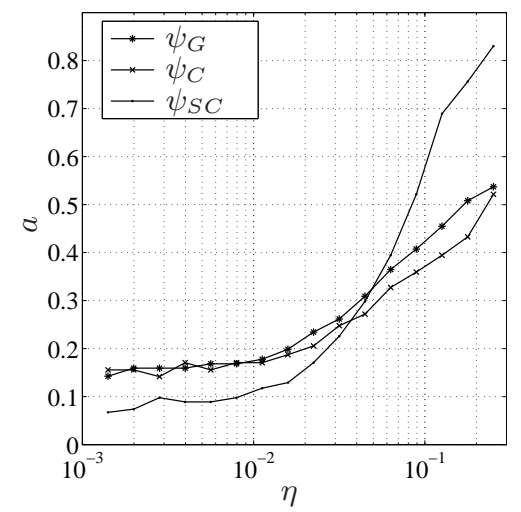

Fig. 3. Optimization result for the slope $a$ as a function of step size $\eta$.

$$
\psi_{S C}\left(y_{i}\right)= \begin{cases}\frac{a^{\prime}}{2}\left(y_{i}+k(M-0.5)+2\right) & y_{i}<-k(M-0.5) \\ a^{\prime} \sin \left(\frac{\pi}{d} y_{i}\right) & \text { otherwise } \\ \frac{a^{\prime}}{2}\left(y_{i}-k(M-0.5)-2\right) & y_{i}>k(M-0.5)\end{cases}
$$

by scaling and shifting the linear functions. As for the previous non-linearities, the scaling $a$ is optimized by simulations (Figure B).

Non-linearities for modulations with discrete points in their constellation diagram can be constructed similarly. For modulations like CPM or FSK the modeling of the pdf will have to be derived via a different approach.

\section{Simulations}

In this section we evaluate the bit error rate (BER) of a zero-IF receiver combined with the proposed separation algorithm and the convergence characteristics of the separation algorithms. The analysis is carried out by Matlab simulations. To classify the performance of the proposed non-linearities, we compare them with the EASI algorithm [6], [10] and three conventional non-linearities:

- Mutual Information (MI) non-linearity [19];

$$
\psi_{M I}\left(y_{i}\right)=\frac{3}{4} y_{i}^{11}+\frac{25}{4} y_{i}^{9}-\frac{14}{3} y_{i}^{7}-\frac{47}{4} y_{i}^{5}+\frac{29}{4} y_{i}^{3},
$$

- Cubic non-linearity [11]; $\psi_{C U}\left(y_{i}\right)=y_{i}^{3}$ and

- Hyperbolic non-linearity [20]; $\psi_{H}\left(y_{i}\right)=y_{i}-\tanh \left(y_{i}\right)$.

We investigate the behavior of these algorithms under the effect of additive white Gaussian noise (AWGN) within baseband. A Gray coded 16-QAM signal which consists of two 4-PAM signals is used for the simulations. The phase and gain error are assumed to be random variables which are uniformly distributed between $\pm 5^{\circ}$ and $\pm 7.5 \%$, respectively (which is a rather severe IQ mismatch). The BER of each algorithm is the result of 50 Monte Carlo runs over these two random variables. Next, the step size $\eta$ is 0.01 for all six II algorithms. Therewith, we get (Fig. B) the values of the slope $a$, namely, 0.17 for the Cauchy and Gaussian non-linearity and 0.11 for the simplified Cauchy non-linearity. The step size of the EASI algorithm is fixed to 0.001 such that the BER of the EASI algorithm and the II algorithm are almost equal. The batch length $L$ is 40 .

Figure 7 shows the resulting BER for typical signal to noise ratios (SNR - $E_{S} / N_{0}$ ). The lower bold line is the BER of 16-QAM receiver without IQ-imbalances. Reaching this BER means complete cancellation of the IQ-imbalances. The upper bold line is the BER which is obtained with an inaccurate receiver and without cancellation of the IQ-imbalances. The gap between the two curves becomes more significant when the SNR is high due to the fact that the IQ-imbalances are then more dominant. We obtain almost the same result for all seven algorithms as intended by the choice of the step size $\eta$. Only the iterative inversion algorithm with the MI non-linearity is sensitive to very low noise. In this case the BER is even worse than the uncancelled signal which is the result of converging to an incorrect separation matrix. Furthermore, the BER degrades with growing step size $\eta$. 


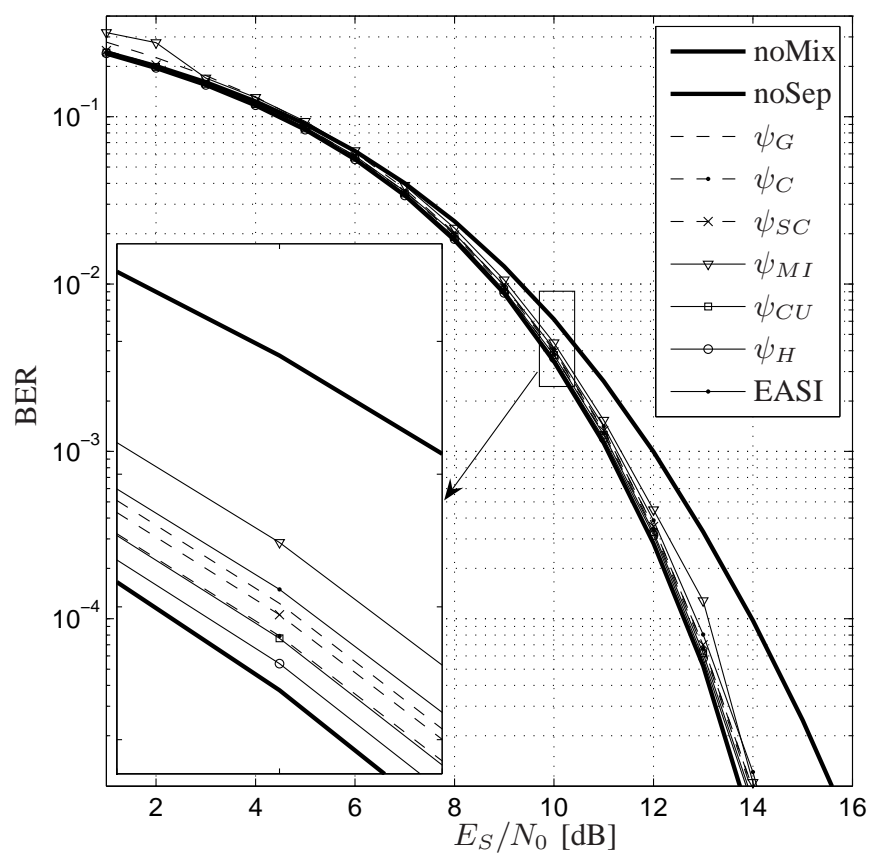

Fig. 4. BER comparison of receiver with pdf dependent $\left(\psi_{G}, \psi_{C}, \psi_{S C}\right)$ and pdf independent II algorithms $\left(\psi_{M I}, \psi_{C U}, \psi_{H}\right)$, with EASI algorithm, without separation (noSep) and without IQ-imbalances (noMix) .

A second property of a BSS-algorithm is the convergence time. To analyze the convergence behavior we use the performance index from equation (10). The convergence time increases at low SNR and decreases with growing step size $\eta$. Hence, the step size can be used to get a tradeoff between BER behavior and convergence time.

The convergence behavior of the algorithm is shown in Figure 5, where we performed 50 Monte Carlo simulations for a SNR of $12 \mathrm{~dB}$, a phase error of $\varphi_{I}=6^{\circ}, \varphi_{Q}=3^{\circ}$ and an amplitude error $g_{I}=1.05, g_{Q}=0.98$.

The semi-blind algorithms outperforms the EASI algorithm and the other three non-linearities. Figure 5(a) illustrates the convergence of the II algorithm with the three pdf dependent non-linearities $\psi_{C}, \psi_{G}, \psi_{S C}$. The initial PI is shown as a dashed line. The zone of convergence can hardly be seen because only around 5 iterations are needed to reach a stable state. Figure 5(b), in contrast, shows the convergence properties of the EASI algorithm and the pdf independent non-linearities $\psi_{M I}, \psi_{C U}$, $\psi_{H}$. The convergence of the algorithm based on pdf dependent non-linearities is much better than with the non-dependent non-linearities. This is a major advantage of our approach.

Indeed, a short convergence time is needed in burst-mode communications, where the IQ-imbalance has to be corrected from the first symbols. To reduce the computational complexity, the separation matrix may be computed only at the start of the burst, under the assumption of slow IQ-imbalance evolution with time.

\section{COnClusions}

In this paper, a new semi-blind source separation algorithm for the cancellation of IQ-imbalances in zero IF-receiver has been introduced. The algorithm is based on an existing iterative inversion blind source separation algorithm, but uses the knowledge of the modulation to significantly enhance its performance.

Compared to the original algorithm, the semi-blind approach performs similarly to the blind one in steady-state. The main advantage of the semi-blind approach is its convergence time, which is very short and makes it suitable in bursty communications. Moreover, similarly to the blind algorithm, no additional bandwidth is necessary which makes the system attractive from the spectral point of view.

This paper considers only IQ-imbalance cancellation for zero-IF receiver but the proposed method is also applicable to lowIF receiver or other receiver architectures based on separate IQ-down-conversion. It is also possible to extend the algorithm to convolutive imbalances caused by the equivalent low pass filter (baseband) transfer function in I- and Q-paths. 


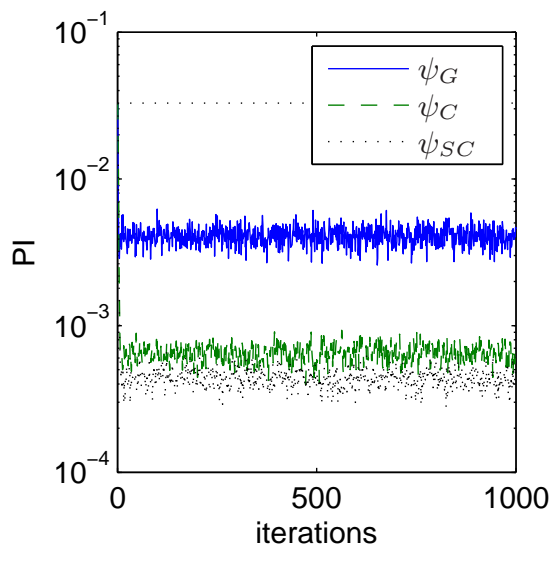

(a) Pdf dependent non-linearities

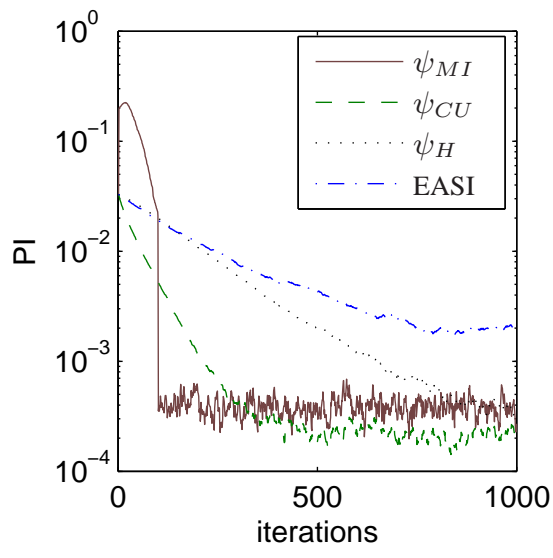

(b) EASI algorithm and universal nonlinearities

Fig. 5. Comparison of the mean convergence behavior for a phase error $\varphi_{I}=6^{\circ}, \varphi_{Q}=3^{\circ}$ and a amplitude error $g_{I}=1.05, g_{Q}=0.98$.

\section{REFERENCES}

[1] T. Hentschel and G. P. Fettweis, CDMA Techniques for Third Generation Mobile Systems, 1999, vol. 489, ch. Software Radio Receivers, pp. $257-283$.

[2] J. Glas, "Digital i/q imbalance compensation in a low-if receiver," IEEE Global Communications Conference, vol. 3, pp. 1461-1466, 1998.

[3] J. Tubbax, B. Cme, L. V. derPerre, L. Deneire, S. Donnay, and M. Engels, "Compensation of iq imbalance in ofdm systems," International Conference on Communications, pp. 3403 - 3407, 2003.

[4] R. Porat and F. Harris, "Resolving and correcting gain and phase mismatch in transmitters and receivers for wideband ofdm systems," Asilomar Conference on Signals, Systems, and Computers, vol. 2, pp. 1005 - 1009, 2002.

[5] M. Windisch and G. Fettweis, "Blind estimation and compensation of receiver i/q imbalance in ofdm systems with enhancements through kalman filtering," Proceedings of the IEEE Workshop on Statistical Signal Processing (SSP'07), p. accepted for publication, 2007.

[6] M. Valkama, M. Renfors, and V. Koivunen, "Advanced methods for i/q imbalance compensation in communication receivers," IEEE Transactions on Signal Processing, vol. 49, pp. 2335 - 2344, 2001.

[7] M. Mailand, R. Richter, and H. Jentschel, "Blind iq-imbalance compensation using iterative inversion for arbitrary direct conversion receivers," IST Mobile \& Wireless Communications Summit, vol. 14, 2005.

[8] M. Valkama, M. Renfors, and V. Koivunen, "Blind signal estimation in conjugate signal models with application to i/q imbalance compensation," IEEE Signal Processing Lett., vol. 12, pp. 733 - 736, 2005.

[9] _ _ "Blind i/q signal separation -based solutions for receiver signal processing," EURASIP Journal on Applied Signal Processing, vol. 16, pp. 2708 2718,2005

[10] J.-F. Cardoso and B. H. Laheld, "Equivariant adaptive source separation,” IEEE Trans. on Signal Processing, vol. 44, no. 12, pp. 3017-3030, 1996.

[11] S. Cruces, A. Cichocki, and L. Castedo-Ribas, "An iterative inversion approach to blind source separation," IEEE Transactions on Neural Networks, vol. 11, no. 6, pp. 1423-1437, 2000.

[12] S. Cruces, "Una visión unificada de los algoritmos de separatión ciega de fuentes," Ph.D. dissertation, Universidad de Vigo, 1999.

[13] J.-F. Cardoso, "Infomax and maximum likelihood for source separation," IEEE Letters on Signal Processing, vol. 4, pp. 112-114, 1997.

[14] D.-T. Pham and P. Garrat, "Blind separation of mixture of independent sources through a quasi-maximum likelihood approach," IEEE Trans. on Signal Processing, vol. 45, no. 7, pp. 1712-1725, 1997.

[15] A. Bell and T. Sejnowski, "An information-maximization approach to blind separation and blind deconvolution," Neural Computation, vol. 7, pp. $1129-1159,1995$.

[16] M. Babaie-Zadeh, C. Jutten, and K. Nayebi, "Differential of the mutual information," Signal Processing Letters, vol. 11, no. 1, pp. 48 - 51, 2004.

[17] S.-I. Amari and J.-F. Cardoso, "Blind source separation—semiparametric statistical approach," IEEE Trans. on Signal Processing, vol. 45, no. 11, pp. 2692-2700, 1997

[18] A. Cichocki and S.-I. Amari, Adaptive Blind Signal and Image Processing. John Wiley \& Sons, 2003.

[19] S.-I. Amari, A. Cichocki, and H. Yang, "A new learning algorithm for blind source separation," in Advances in Neural Information Processing Systems 8. MIT Press, 1996, pp. 757-763.

[20] M. Girolami, "An alternative perspective on adaptive independent component analysis algorithms," Neural Computation, vol. 10, no. 8, pp. 2103-2114, 1998. [Online]. Available: http://cis.paisley.ac.uk/research/reports/index.htm] 